\title{
THE SCHOTTKY PROBLEM ON PANTS
}

\author{
R. C. PENNER
}

(Communicated by Irwin Kra)

\begin{abstract}
In this note, we consider the classical problem of Schottky of characterizing the set of period matrices which arise from all possible conformal structures on a fixed topological surface. Restricting to a planar surface with Euler characteristic -1 , we find that a real symmetric 3 -by-3 matrix arises as a period matrix if and only if the matrix has vanishing row sums, and the diagonal entries are positive and satisfy all three possible strict triangle inequalities. The technique of proof involves extremal and harmonic lengths of curve classes.
\end{abstract}

In this short note, we describe a solution to the Schottky problem for "pairs of pants" (i.e., connected planar surfaces with Euler characteristic -1). This work grew out of an attempt several years ago to compute period matrices by decomposing a conformal surface of negative Euler characteristic into pairs of pants, as is so effective in the hyperbolic category. Such a program would require a (numerical) conformal invariant of homotopy classes of curves in a pants decomposition and a complete analysis of pairs of pants. This latter analysis is given herein. The results of this note do not seem to follow directly from the substantial $K-P$ machinery of [AdC, Mu, Sa, SW, Sh]. Indeed, our techniques are pedestrian manipulations of extremal and harmonic lengths.

It is a pleasure to thank the visitors to Institut Mittag-Leffler during 1983-1984, especially Tapani Kuusalo and Allen Weitsman, for helpful discussion.

Let $P$ denote the closed disc minus two interior open discs, or pair of pants, and let $\partial_{i}, i=1,2,3$, denote the components of $\partial P$. The Teichmüller space $\mathscr{T}$ of $P$ (consisting of marked conformal structures on $P$ ) is a three (real)-dimensional ball. Indeed, each marked conformal structure $\rho$ (i.e., marked conformal class of finitearea complete metric with geodesic boundary) is represented by a unique hyperbolic (intrinsic Poincaré) metric $\mu \in \rho$; these latter are in one-to-one correspondence with $\mathbf{R}_{+}^{3}$, via the map $\mathscr{T} \rightarrow \mathbf{R}_{+}^{3}, \rho \rightarrow\left(l_{i}(\mu)\right)_{1}^{3}$, where $l_{i}(\mu)$ is the $\mu$-length of $\partial_{i}$ (see, e.g., [Th, FLP]). In this note, we give a conformal analogue of this basic fact from hyperbolic geometry.

If $\rho \in \mathscr{T}$, let $U(\rho)$ denote the collection of functions $0<u<1$ harmonic on $P$ with respect to $\rho$, and define the harmonic length (see [LO]) of $\partial_{i}$ for $\rho$ to be

$$
h_{i}(\rho)=\sup \int_{\partial i} * d u
$$

Received by the editors April 9, 1987 and, in revised form, October 5, 1987.

1980 Mathematics Subject Classification (1985 Revision). Primary 30C20, 30F20, 53A30, 58C10; Secondary 30F 15, 58B20.

Partially supported by the N.S.F. 
where the sup is over all $u \in U(\rho)$ and $*$ denotes harmonic conjugate. Our main result is

THEOREM. A triple $\left(h_{i}\right)_{1}^{3}$ occurs as $\left(h_{i}(\rho)\right)_{1}^{3}$ for some $\rho \in \mathscr{T}$ if and only if

$$
h_{i}<h_{j}+h_{k}, \quad \text { for }\{i, j, k\}=\{1,2,3\} \text {. }
$$

Moreover, such an $\left(h_{i}\right)_{1}^{3}$ uniquely determines $\rho \in \mathscr{T}$.

To begin, we have

LEMMA 1. The strict triangle inequalities $(*)$ are necessary for $\left(h_{i}\right)_{1}^{3}$ to arise as $\left(h_{i}(\rho)\right)_{1}^{3}$ for some $\rho \in \mathscr{T}$.

PROOF. Let $w_{1}^{\rho}$ denote the harmonic measure of $\partial_{i}$ for $\rho$, so that $w_{i}^{\rho}$ solves the Dirichlet problem on $P$ with boundary values $w_{i}^{\rho}=1$ on $\partial_{i}, w_{i}^{\rho}=0$ on $\partial P-\partial_{i}$. Fix $\rho \in \mathscr{T}$, and set $w_{i}=w_{i}^{\rho}, i=1,2,3$. We concentrate on the inequality $h_{1}<h_{2}+h_{3}$, the others being analogous. Since $\int_{\partial P} * d w_{1}=0$, we have $h_{1} \leq h_{2}+h_{3}$ with equality only if

$$
\int_{\partial_{i}} * d w_{i}=\int_{\partial_{i}} * d w_{1}, \quad i=2,3
$$

Finally, $* d w_{1}+* d w_{2}+* d w_{3}=0$, whence

$$
\int_{\partial_{3}} * d w_{2}=\int_{\partial_{2}} * d w_{3}=0
$$

which is absurd.

To proceed, we define the conjugate extremal length (see [A2]) of $\partial_{i}$ for $\rho$ to be

$$
\lambda_{i}(\rho)=\sup \frac{l_{i}^{2}(\mu)}{[\mu \text {-area of } P]}
$$

where the sup is over all finite-area metrics $\mu$ representing $\rho$, and $l_{i}(\mu)$ is the infimum of $\mu$-lengths of curves separating $\partial_{i}$ from $\partial P-\partial_{i}$.

LEMMA 2. For $\rho \in \mathscr{T}$, we have

$$
h_{i}(\rho)=\int_{\partial_{i}} * d w_{i}^{\rho}=\lambda_{i}(\rho)
$$

PROOF. Theorem 4.5 of [A2] guarantees the second equality (which we do not need below), and we concentrate on the first in case $i=1$. The proof follows that of [LO, Theorem 2.1] and is included for completeness. Let $w_{1}=w_{1}^{\rho}$ and suppose $v \in U(\rho)$ so that $w_{1} * d v-v * d w_{1}$ is a closed one-form on $P$. We find

$$
\begin{aligned}
\int_{\partial_{1}} * d v & =\int_{\partial P} v * d w_{1}=-\int_{\partial P} v \frac{\partial w_{1}}{\partial n} d s \\
& =-\int_{\partial_{1}} v \frac{\partial w_{1}}{\partial n} d s+\int_{\partial P-\partial_{1}} v \frac{\partial w_{1}}{\partial n} d s \\
& \leq \int_{\partial_{1}} * d w_{1}
\end{aligned}
$$

since $0<v<1$ and $\partial w_{1} / \partial n<0$ on $\partial P-\partial_{i}$.

To show the triangle inequalities $(*)$ are sufficient, we must construct $\rho \in \mathscr{T}$ from a putative triple $\left(h_{i}\right)_{1}^{3}$. It is convenient to uniformize $\rho \in \mathscr{T}$ as a "slit annulus" (see, 
e.g., $[\mathbf{A 1}])$, so $\rho \in \mathscr{T}$ is described by parameters $(R, r, \theta)$, where $(P, \rho)$ is conformal to a flat annulus in $\mathbf{C}$ of inner boundary component $\{|z|=1\}$ and outer boundary component $\{|z|=R\}$ with a connected proper subset (the "slit") of $\{|z|=r<R\}$ removed. The parameter $0<\theta<2 \pi$ gives the angle from $0 \in \mathbf{C}$ subtended by the slit. Such a uniformization is accomplished by considering the period matrix.

$$
A=A(\rho)=\left(\alpha_{i j}\right)_{i, j=1,2,3}
$$

of $\rho \in \mathscr{T}$, where

$$
\alpha_{i j}=\alpha_{i j}(\rho)=\int_{\partial_{i}} * d w_{j}=\int_{\partial_{j}} * d w_{i} .
$$

Remark that $h_{i}=h_{i}(\rho)=\alpha_{i i}(\rho)$ by Lemma 2 .

A nice fact about the pair of pants is that the diagonal $\left(h_{i}\right)_{1}^{3}$ completely determines the period matrix. Indeed, $A$ is symmetric and each row-sum vanishes. Solving a linear system then gives

$$
\alpha_{i j}=\frac{h_{k}-h_{i}-h_{j}}{2}, \quad \text { for }\{i, j, k\}=\{1,2,3\} .
$$

In particular, the triangle inequalities $(*)$ guarantee that off-diagonal entries of $A$ are negative (as one would expect).

As in [A 1, pp. $199 \mathrm{ff}$.], the radii $R, r$ are easily computed from $A$. Indeed, if we wish to map $\partial_{3}$ to $\{|z|=1\}, \partial_{1}$ to $\left\{|z|=e^{t_{1}}\right\}$ and $\partial_{2}$ to the slit contained in $\left\{|z|=e^{t_{2}}\right\}, t_{1}>t_{2}$, then we have

$$
\left[\begin{array}{cc}
h_{1} & \alpha_{12} \\
\alpha_{12} & h_{2}
\end{array}\right]\left[\begin{array}{l}
t_{1} \\
t_{2}
\end{array}\right]=\left[\begin{array}{c}
2 \pi \\
0
\end{array}\right]
$$

and find

$$
\left[\begin{array}{l}
t_{1} \\
t_{2}
\end{array}\right]=\frac{1}{\delta}\left[\begin{array}{cc}
h_{2} & -\alpha_{12} \\
-\alpha_{12} & h_{1}
\end{array}\right]\left[\begin{array}{c}
2 \pi \\
0
\end{array}\right]=\frac{2 \pi}{\delta}\left[\begin{array}{c}
h_{2} \\
-\alpha_{12}
\end{array}\right]
$$

where

$$
\delta=h_{1} h_{2}-\alpha_{12}^{2}=-\frac{1}{2}\left(h_{1} \alpha_{23}+h_{2} \alpha_{13}+h_{3} \alpha_{12}\right)>0 .
$$

Instead of trying to compute the slit parameter $\theta$, we uniformize another way mapping $\partial_{1}$ to $\{|z|=1\}, \partial_{2}$ to $\left\{z=e^{\tau}\right\}$, and $\partial_{3}$ to the slit. As above, one computes $\tau=2 \pi h_{3} / \delta$, and arithmetic gives

$$
h_{1}=\frac{\delta}{2 \pi}\left(2 t_{2}-t_{1}+\tau\right), \quad h_{2}=\frac{\delta}{2 \pi} t_{1}, \quad h_{3}=\frac{\delta}{2 \pi} \tau
$$

and

$$
\delta=4 \pi^{2} /\left(\tau t_{1}-\left(t_{1}-t_{2}\right)^{2}\right) .
$$

Thus, the transformation between $\left(h_{i}\right)_{1}^{3}$ and $\left(t_{1}, t_{2}, \tau\right)$ is bijective, and it remains only to understand the sense in which $\left(t_{1}, t_{2}, \tau\right)$ coordinatize $\mathscr{T}$.

LEMMA 3. The marked conformal structure of a slit annulus with inner radius 1 is uniquely determined by: the outer radius $R$; the radius $r$ of the slit; the conjugate extremal length $\lambda$ of connected curves separating the slit from the outer boundary. Moreover, a triple $(R, r, \lambda)$ arises if and only if

$$
\log r+2 \pi \lambda^{-1}>\log R>\log r>0 .
$$


PROOF. We claim that $\lambda$ is a monotone increasing function of angular slit size $\theta$. To see this, consider another uniformization as a slit annulus where the slit and inner boundary are interchanged, and let $e^{\tau}$ denote the new outer radius. Clearly $\lambda=2 \pi \tau^{-1}$, and monotonicity of $\tau$ (and hence $\lambda$ ) follows from monotonicity of the Grötsch map (see [LV, II§2]).

Now, the limiting cases occur as $\theta \rightarrow 0$ when $\lambda \rightarrow 0$ and as $\theta \rightarrow 2 \pi$ when $\lambda \rightarrow 2 \pi / \log \left(R r^{-1}\right)$; a continuity argument finishes the proof.

We conclude the proof of the main theorem by identifying $t_{1}=\log R, t_{2}=\log r$, $\tau=2 \pi \lambda^{-1}$, and checking that the inequalities $(* *)$ are equivalent to the triangle inequalities $(*)$.

COROLlaRY. A 3-by-3 matrix $A$ over $\mathbf{R}$ is the period matrix of a pair of pants if and only if $A$ is symmetric, has vanishing row-sums, and the diagonal entries are positive and satisfy all possible strict triangle inequalities.

\section{BIBLIOGRAPHY}

[A1] L. V. Ahlfors, Complex analysis, McGraw-Hill, 1953.

[A2] _ Conformal invariants, McGraw-Hill, 1973.

[AdC] E. Arbarello and C. de Concini, On a set of equations characterizing Riemann matrices, Ann. of Math. (2) 120 (1984), 119-140.

[FLP] A. Fathi, F. Laudenbach, V. Poenaru, et al., Travaux de Thurston sur les surfaces, Asterisque 30 (1979), 66-67.

[LO] H. J. Landau and R. Osserman, On analytic mappings of Riemann surfaces, J. Analyse Math. 7 (1959), 249-279.

[Mu] M. Mulase, Cohomological structure in soliton equations and Jacobian varieties, J. Differential Geom. 19 (1984), 403-430.

[LV] O. Lehto and K. I. Virtanen, Quasiconformal mappings in the plane, Springer-Verlag, 1973.

[Sa] M. Sato and Y. Sato, Soliton equations as dynamical systems on infinite dimensional Grassmann manifolds, preprint.

[Sh] T. Shiota, Characterization of Jacobian varieties in terms of soliton equations, Harvard Univ., preprint.

[SW] G. Segal and G. Wilson, Loop groups and equations of KdV type, Publ. Inst. Hautes Études Sci. 61 (1985), 5-65.

[Th] W. P. Thurston, The geometry and topology of three-manifolds, Princeton Univ. Lecture Notes, 1979.

Department of Mathematics, University of Southern California, Los AnGELES, CALIFORNIA 90089 